\title{
Fenômeno de Will Rogers: \\ O Que Todo Médico Deve Saber
}

\author{
José Mauricio Mota, MD, PhD 1, 2, 3 *; \\ Paulo M. Hoff, MD, PhD 1, 2, 4, 5 \\ Afiliaçóes: \\ 1. Instituto do Câncer do Estado de São Paulo, Universidade de São Paulo, São Paulo, Brasil \\ 2. Instituto D'Or de Pesquisa e Ensino, São Paulo, Brasil \\ 3. Programa Jovens Lideranças Médicas, Academia Nacional de Medicina, Brasil \\ 4. Academia Nacional de Medicina, Brasil

\section{Autor-Correspondente:} \\ José Mauricio Mota, Av. Dr. Arnaldo, 251, CEP: 01246-000, São Paulo, SP, Brasil. \\ (11)3893-2000. Email: jose.mota@hc.fm.usp.br \\ Palavras-chave: fenômeno de Will Rogers, migração de estágio, viés \\ Conflitos de interesse: nenhum a declarar.
}

William Penn Adair Rogers, ou simplesmente Will Rogers, foi um showman norte-americano que viveu entre $1879 \mathrm{e}$ 1935. Nos dias de hoje, seu nome é pouco conhecido, mas Will Rogers foi muito famoso em seu tempo. Sua morte prematura após um trágico acidente aéreo rendeulhe várias homenagens e memoriais em solo americano. Curiosamente, chegou a morar nos pampas argentinos durante sua juventude, seguindo o estilo de vida e de trabalho gaúcho por um breve período.

Will Rogers era conhecido pela irreverência e talento cômico, brincando com tudo o que estava a seu alcance. Vivendo o período da grande depressão econômica do início da década de 1930, observou a intensa migração de pessoas das cidades do interior para as grandes capitais. O próprio Rogers nasceu em Oklahoma (mais precisamente em território da Nação Cherokee) e mudou-se para a California após tornar-se uma estrela de Hollywood. É atribuída a ele a frase: "When the Okies left Oklahoma and moved to California, they raised the average intelligence level in both states" [em tradução livre: quando os naturais de Oklahoma saíram de lá e mudaram-se para a California, eles aumentaram a média do nível de inteligência nos dois estados]. A conclusão de Rogers parte de três premissas fundamentais, que estão implícitas em sua 
piada: (i) a média de inteligência entre os californianos seria menor que a média da inteligência das pessoas de Oklahoma; (ii) as pessoas de Oklahoma que migram teriam menor inteligência comparada ao seu grupo de origem; e (iii) as pessoas de Oklahoma que migram teriam maior inteligência comparada aos californianos. Mas qual a relação disso tudo com a Medicina?

Will Rogers, talvez sem imaginar o impacto de sua inocente piada, trouxe importantes reflexôes para pesquisadores mé- dicos, bioestastísticos e epidemiologistas. Primeiro é importante entender a plausibilidade matemática desse fenômeno de mudança de médias para a mesma direçáo em dois agrupamentos de dados diferentes. No exemplo da Figura 1, temos dois conjuntos: "A" e "B". A média dos dados em "A" é igual a 2 e a média dos dados em "B" é igual a 5 . Se a variável 4 for transportada para o conjunto "A", teremos uma nova situação em que as médias são 2,5 e 5,5, respectivamente. Esse fenômeno pode acontecer na

\section{Cenário 1}

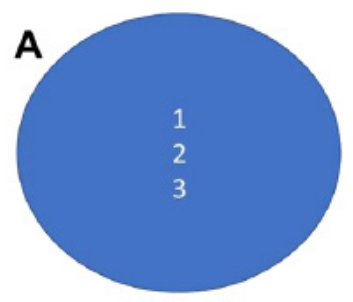

Média $=2$

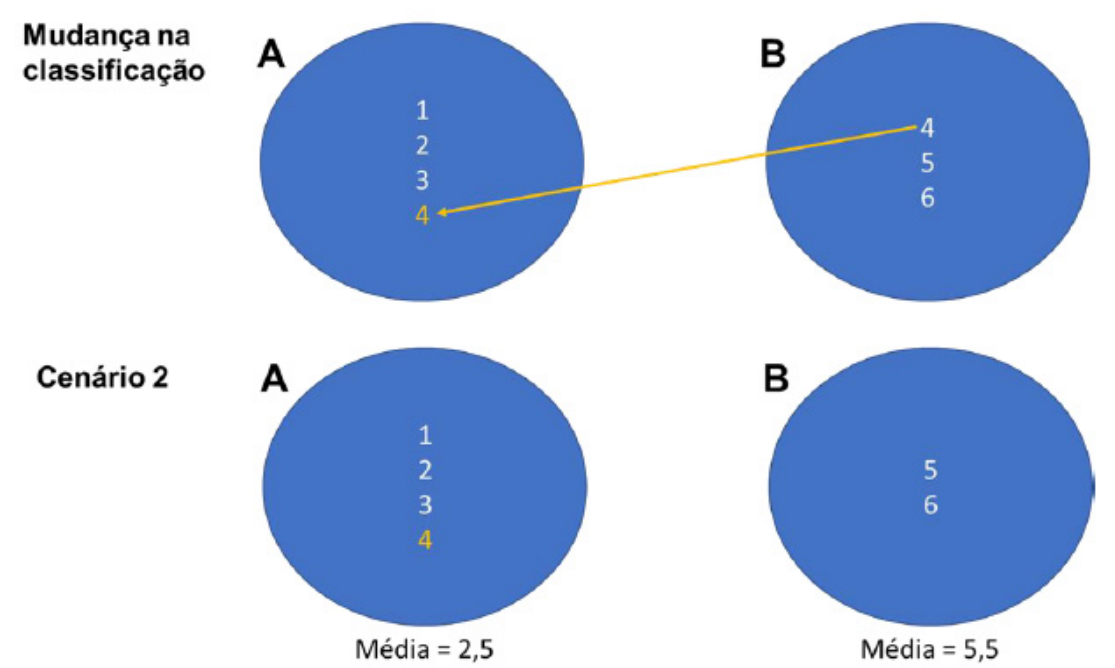

Figura 1: Fenômeno de Will Rogers. A transposição do menor valor do conjunto "B" para o conjunto "A" acarreta a variação de médias para cima nos dois grupos. 
Medicina, modificando artificialmente desfechos de acordo com mudanças na classificação diagnóstica de pacientes, como abaixo descrevemos.

Apesar de evidências anteriores já terem sugerido a ocorrência desse fenômeno em estudos epidemiológicos, ${ }^{1}$ foi apenas em 1985 que Alvan Feinstein e co-autores cunharam o termo "Fenômeno de Will Rogers" para descrever o viés resultante de mudanças na classificação diagnóstica. 2 Nesse artigo, duas coortes de pacientes com câncer de pulmão foram analisadas: pacientes tratados em $1977(\mathrm{~N}=131)$ e pacientes tratados entre 1953 e $1964(\mathrm{~N}=1266)$. Os autores notaram um aumento de sobrevida global em todos os subgrupos da coorte mais recente quando comparada à mais antiga. Mantendo um saudável ceticismo necessário à boa ciência, os autores não atribuíram simplesmente seus resultados a avanços médicos do tempo mais moderno, como por exemplo, o uso de esteróides, antibióticos, e cuidados hospitalares em geral. Ao contrário, os autores demonstraram que o aumento observado na sobrevida desses pacientes era irreal, um mero artefato secundário a mudanças na classificação dos pacientes antes considerados como não-metastáticos para metastáticos. Esse fenômeno de migraçáo de estágio ocorreu em decorrência do uso de métodos radiológicos mais sensíveis para detectar doença metastática. Na coorte de 1977 , os pacientes que poderiam ter sido classificados como pertencentes a uma fase inicial da doença, foram mais frequentemente designados para classificaçóes mais avançadas por esse motivo. Isso levou ao fenômeno de mudança de médias que descrevemos acima, produzindo artificialmente melhora das taxas de sobrevida em todos os subgrupos da coorte de 1977, quando comparada a coorte de 1953 a 1964. Esse achado ficou evidente após os autores demonstrarem que as taxas de sobrevida entre as coortes foi semelhante quando os pacientes eram classificados de acordo com um método de estadiamento baseado apenas em sintomas. ${ }^{2}$

A partir de então, o fenômeno de Will Rogers foi demonstrado em várias outras situaçôes clínicas, como por exemplo em estudos de câncer de próstata,34 câncer colorretal, ${ }^{5}$ câncer de esôfago 6 e esclerose múltipla. ${ }^{7}$ Talvez o câncer de próstata seja uma das doenças em que deveríamos ter uma atençáo redobrada para a possibilidade desse tipo de viés produzido pela migração de estágio, haja vista a ocorrência de várias modificaçóes e aperfeiçoamentos na classificação desses pacientes ao longo das últimas décadas. ${ }^{8,9}$

Um dos exemplos mais notáveis de mudança de classificação em câncer de próstata que ocorreu ao longo dos anos foi a exclusão dos adenocarcinomas classificados anteriormente como Gleason 2, 3 ou 4. Por sugestão do Dr. Jonathan Epstein, um dos pioneiros na classificação de risco de 
pacientes com câncer de próstata, os tumores previamente reportados como Gleason 2 a 4 não deveriam ser diagnosticados como câncer. ${ }^{10}$ Essa sugestão possivelmente influenciou resultados de sobrevida devido ao fenômeno de Will Rogers. Em um trabalho publicado em 2005, foi realizada a re-leitura entre os anos de 2002 e 2004 de biópsias de próstata realizadas entre 1990 e 1992. Os autores demonstraram que 55\% das biópsias tiveram aumentos do escore de Gleason, enquanto apenas $14 \%$ tiveram reduçóes, ou seja, indivíduos previamente classificados como tendo doença de mais baixo risco, foram re-classificados como tendo doença de risco maior. Devido ao fenômeno de Will Rogers, a sobrevida em cada um dos escores de Gleason foi maior quando seguiuse a classificação de 1990 a 1992.(BJU Int. 2005; 95: 1146-1152)

O emprego de técnicas de tomografia por emissão de pósitrons e novos radiotraçadores mais sensíveis e específicos para detecção do câncer de próstata, como o Gálio-68-PSMA-617 ("PET de PSMA"), ${ }^{11,12}$ também tem trazido à tona a possibilidade de observarmos uma melhora espúria na sobrevida dos pacientes com câncer de prós-

\section{Exames convencionais}

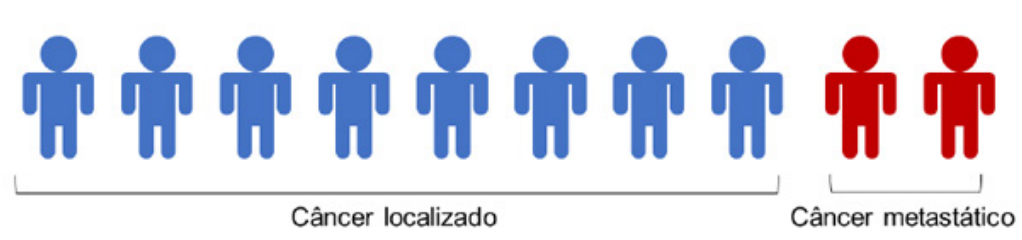

\section{PSMA PET/TC}

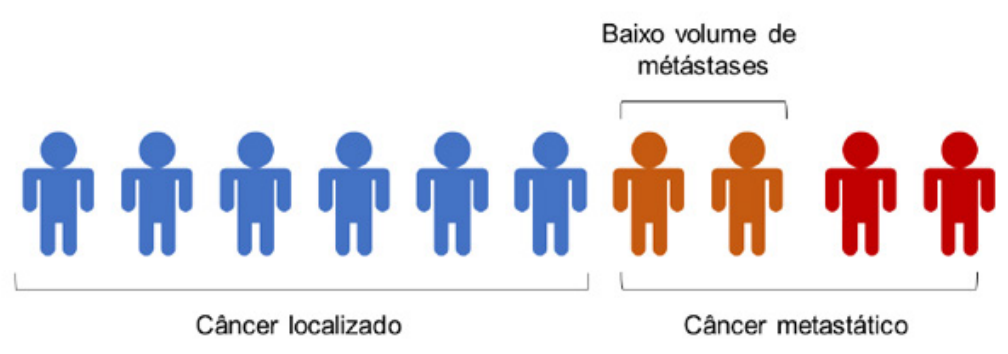

Figura 2. Fenômeno de Will Rogers e o emprego de imagem molecular em pacientes com câncer de próstata. O emprego de métodos mais sensiveis e especificos de imagem, como o PSMA PET/TC, identifica metástases em pacientes previamente classificados como tendo doença localizada, levando à migração de estágio. Os pacientes que passam por essa classificação mais provavelmente têm um baixo volume de doença metastática, tendo, portanto, possivelmente melhor prognóstico que o grupo para o qual foram transportados ("câncer metastático") e pior prognóstico que o grupo de origem ("câncer localizado"). Exames convencionais: cintilografia óssea, tomografia computadorizada e ressonância magnética. PET/TC: tomografia por emissão de pósitrons com tomografia computadorizada; PSMA: antígeno de membrana prostático especifico. 
tata. Na prática, o PET de PSMA tem sido utilizado principalmente para o estadiamento inicial de pacientes com câncer de próstata e detecção de doença metastática em pacientes com recorrência bioquímica (elevação do PSA) após tratamento do tumor primário. Nesses cenários, fica claro que o emprego de um método mais sensível e específico para detectar doença metastática pode produzir a reclassificação de muitos pacientes: homens que seriam anteriormente classificados como tendo doença localizada ou recorrência bioquímica, podem ser redesignados para o grupo de indivíduos com doença metastática.

Pelo fato desses pacientes serem os "menos graves" desse último grupo, essa mudança de classificação poderá levar a observações populacionais de aumento de sobrevida, sem, contudo, interferir na sobrevida individual do paciente (Figura 2). De fato, ainda não compreendemos qual o verdadeiro papel do PET de PSMA nesses pacientes com câncer de próstata. Por outro lado, evidências preliminares sugerem que o tratamento precoce da doença oligometastática pode trazer benefícios para os pacientes $^{13,14}$, assim como o tratamento direcionado para os achados de imagem molecular poderia melhorar desfechos em pacientes com recorrência bioquímica. ${ }^{15}$

Em resumo, o fenômeno de Will Rogers deve ser lembrado como um tipo de viés que pode interferir na interpretação de estudos clínicos. Sempre que observarmos melhoras de desfechos ao longo do tempo, lembremo-nos de que esse achado pode ser espúrio, um efeito produzido pela mudança de classificação ou estadiamento desses indivíduos ao longo do tempo. Em última análise, devemos nos manter céticos quando observarmos ganhos de sobrevida em um dado grupo de pacientes. A solução para esse problema estatístico não é fácil, mas existem estratégias que podem ser utilizadas para minimizá-lo.16. Mesmo anos depois do falecimento de Will Rogers, a irreverente frase a ele atribuída ("When the Okies left Oklahoma and moved to California, they raised the average intelligence level in both states") continua nos ajudando a interpretar melhor os resultados de estudos clínicos em benefício dos nossos pacientes.

\section{REFERÊNCIAS}

1. Trasti H, Nilsson S, Peterson L-E: Applied Diagnostic Techniques:

A Decisive Factor in the Longterm T-year Survival Rate in Prostatic Carcinoma. Br J Urol. 1979;51(2):135-139.

2. Feinstein AR, Sosin DM, Wells CK: The Will Rogers Phenomenon. N Engl J Med. 1985;312(25):1604-1608.

3. Lee WR: Improvement in Prostate Cancer Survival Over Time. Cancer J. 
2012;18(1):9-10.

4. Albertsen PC, Hanley JA, Barrows $\mathrm{GH}$, et al: Prostate Cancer and the Will Rogers Phenomenon. JNCI J Natl Cancer Inst. 2005;97(17):12481253.

5. Shahrier M, Ahnen DJ: Colorectal cancer survival in Europe: the Will Rogers phenomenon revisited. Gut. 2000;47(4):463-464. doi:10.1136/ gut.47.4.463

6. Pietrzak AK, Martenka P, Strzesak E, et al: Will Rogers phenomenon in the oesophageal cancer patients staging - CT versus 18F-FDG PET/CT: retrospective study. Hell J Nucl Med. 22 Suppl 2:174-180.

7. Pia Sormani M: The Will Rogers phenomenon: the effect of different diagnostic criteria. J Neurol Sci. 2009;287:S46-S49.

8. Scher HI, Halabi S, Tannock I, et al: Design and end points of clinical trials for patients with progressive prostate cancer and castrate levels of testosterone: recommendations of the Prostate Cancer Clinical Trials Working Group. J Clin Oncol. 2008;26(7):1148-1159.

9. Scher HI, Morris MJ, Stadler WM, et al: Trial design and objectives for castration-resistant prostate cancer: Updated recommendations from the prostate cancer clinical trials working group 3. J Clin Oncol. 2016;34(12):1402-1418.

10. Epstein JI: Gleason Score 2-4 Adenocarcinoma of the Prostate on Needle Biopsy. Am J Surg Pathol. 2000;24(4):477-478.

11. Hofman MS, Lawrentschuk N, Francis RJ, et al: Prostate-specific membrane antigen PET-CT in patients with high-risk prostate cancer before curative-intent surgery or radiotherapy (proPSMA): a prospective, randomised, multicentre study. Lancet (London, England). 2020;395(10231):1208-1216.

12. Evans JD, Jethwa KR, Ost $P$, et al: Prostate cancer-specific PET radiotracers: A review on the clinical utility in recurrent disease. Pract Radiat Oncol. 2018;8(1):28-39.

13. Ost P, Reynders D, Decaestecker K, et al: Surveillance or Metastasis-Directed Therapy for Oligometastatic Prostate Cancer Recurrence: A Prospective, Randomized, Multicenter Phase II Trial. J Clin Oncol. 2018;36(5):446453.

14. Phillips R, Shi WY, Deek M, et al: Outcomes of Observation vs Stereotactic Ablative Radiation for Oligometastatic Prostate Cancer: The ORIOLE Phase 2 Randomized Clinical Trial. JAMA Oncol. 2020;6(5):650-659. 
15. Jani A, Schreibmann E, Goyal S, et al: Initial Report of a Randomized Trial Comparing Conventional- vs Conventional plus Fluciclovine (18F) PET/CT Imaging-Guided Post-Prostatectomy Radiotherapy for Prostate Cancer. Int J Radiat Oncol. 2020;108(5):1397.

16. Stander M, Stander J: A simple method for correcting for the Will Rogers phenomenon with biometrical applications. Biometrical J. 2020;62(4):1080-1089. 